\title{
Aspects cytogénétiques de l'ataxie télangiectasie
}

L'ataxie télangiectasie se situe à un carrefour où se croisent l'immunologie, la cancérologie, la cytogénétique et la génétique moléculaire. Elle constitue ainsi une maladie modèle pour l'étude des processus de cancérisation.

\section{Alain Aurias \\ Chargé de recherches à l'Inserm, Institut Curie}

\section{ADRESSE ET TIRES A PART}

A. Aurias : Institut Curie, section de biologie, 26, rue d'Ulm, 7523I Paris Cedex 05. 'ataxie télangiectasie (AT) est une affection génétique récessive autosomique dont l'incidence est estimée à un cas pour 40000 naissances. Cliniquement, cette maladie associe une ataxie cérébelleuse, des télangiectasies cutanéomuqueuses et des infections à répétition liées à un déficit immunitaire souvent sévère. Le pronostic en est extrêmement sombre puisque l'espérance moyenne de vie n'est que de seize ans. Cette évolution très défavorable est dominée par la récurrence des infections respiratoires et la survenue de cancers, surtout lymphoïdes, chez plus de $10 \%$ des patients. Ces enfants présentent en outre une très grande radiosensibilité, ce qui rend délicat l'usage de la radiothérapie et des drogues radiomimétiques pour le traitement de leurs cancers. Enfin, cette maladie s'accompagne de cassures et de remaniements chromosomoqies très caractéristiques qui permettent d'en confirmer le diagnostic et, dans une certaine mesure, d'en préciser le pronostic oncologique. Le déterminisme génétique, le risque élevé de cancers, la radiosensibilité exagérée et les anomalies cytogénétiques permettent de classer cette affection dans le groupe des maladies cassantes* précancéreuses, comme le syndrome de Bloom et l'anémie de Fanconi. Dans cet article, nous nous intéresserons aux aspects cytogénétiques de l'ataxie télangiectasie.

\section{Instabilité chromosomique}

C'est en I966 que Hecht et coll. [I] mettaient l'accent sur l'instabilité chromosomique observée dans les cellules des patients AT. Cette instabilité chromosomique, caractérisée par une augmentation de la fréquence des cassures, lacunes, images radiales, etc. a été largement confirmée par de nombreuses études ultérieures. Cependant, ce paramètre reste difficile à étudier car cette instabilité chromosomique est très variable d'un patient à l'autre, voire chez le même patient d'un examen à l'autre. Par ailleurs, ces aberrations spontanées qui peuvent toucher n'importe quel chromosome ne sont pas spécifiques de l'ataxie télangiectasie et sont retrouvées dans des situations pathologiques variées.

L'utilisation en routine des techniques de marquage en bandes des chromosomes allait permettre, à partir des années 70, de constater 


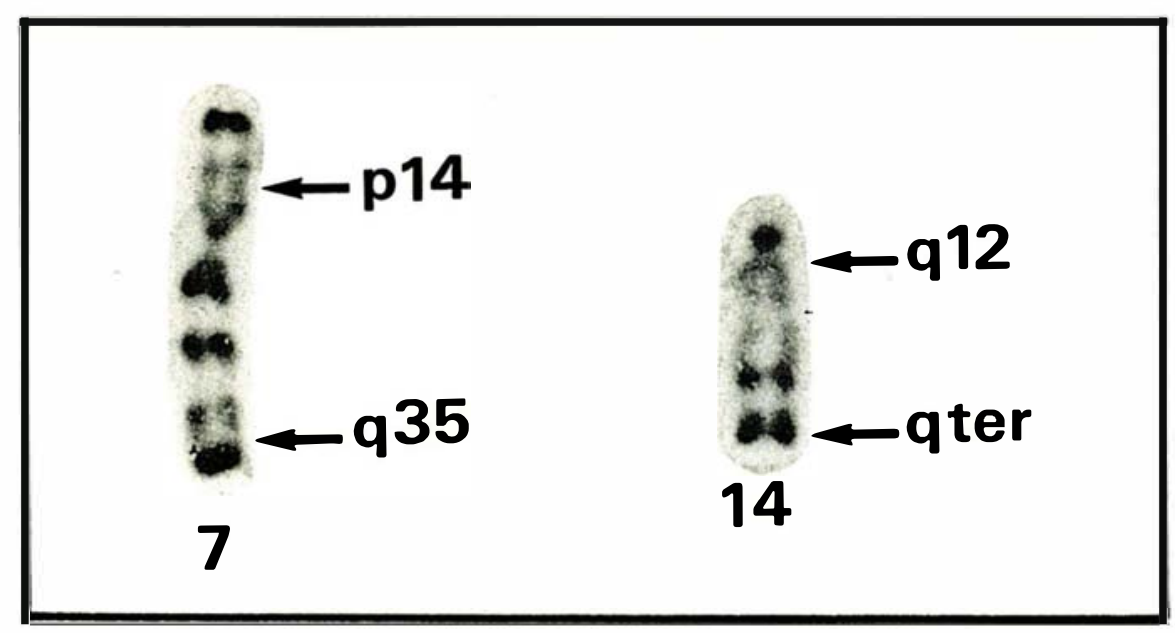

Figure 1. Localisation des bandes du chromosome 7 et du chromosome 14 impliquées dans les remaniements sporadiques récurrents.

dans les lymphocýtes des patients AT une grande fréquence de remaniements chromosomiques stables : translocations* réciproques et inversions de segments de chromosomes [2]. Il apparait maintenant clairement que l'on peut distinguer trois stades successifs dans la survenue de ces remaniements.

\section{Premier stade évolutif}

Cette première étape est commune à tous les patients AT. A ce stade, l'analyse cytogénétique des lym- phocytes périphériques stimulés par la phytohémagglutinine (PHA) ou le pokemeed mitogen (PWM) permet de déceler en moyenne 10\% de mitoses porteuses d'un remaniement chromosomique [3]. Un fait beaucoup plus intéressant est que $60 \%$ de ces remaniements n'impliquent que les chromosomes 7 et/ou I4, en des points très précis qui sont les bandes 7pi4*, 7935, I4qI2 et I 4qter (figure I). Les huit types de remaniements possibles (figure 2, p. suivante) sont, par ordre de fréquence, des inversions péricentriques* du chromosome 7, des trans-
Tableau I

LOCALISATIONS CHROMOSOMIQUES DES GENES DE LA SUPERFAMILLE DES IMMUNOGLOBULINES

$\begin{array}{llll}\text { Ig kappa } & 2 \mathrm{p} 11 & \text { TCR } \alpha & 14 \mathrm{q} 11.2 \\ \text { Leu2/T8 } & 2 \mathrm{p} 12 & \text { IgH } & 14 \mathrm{qter} \\ \text { TCR } \beta & 7 \mathrm{q} 35 & \text { Ig lambda } & 22 \mathrm{q} 11.2 \\ \text { TCR } \gamma & 7 \mathrm{p} 14 & & \end{array}$

IgH : chaîne lourde des immunoglobulines; Ig kappa et lambda : chaînes légères kappa et lambda des immunoglobulines; Leu2/T8: antigènes de différenciation lymphocytaires; TCR : $T$ cell receptor, récepteur des cellules $T$. locations entre un chromosome 7 et un chromosome 14 , des translocations entre les deux chromosomes 7 , des inversions du chromosome 14 et des translocations entre les deux chromosomes I 4 . Il est à noter que tous ces remaniements peuvent être observés chez un même patient et que leur fréquence est indépendante du mode de stimulation des lymphocytes (PHA ou PWM) et de la durée de la culture, ce qui est en faveur de la survenue de ces remaniements in vivo.

A côté de ces remaniements extrêmement caractéristiques et dont la fréquence permet de porter avec une assez grande certitude le diagnostic d'ataxie télangiectasie, on observe environ $0,5 \%$ de mitoses porteuses d'une translocation impliquant l'une des bandes 7pI4, 7935, I4qI 2 ou I 4qter et une autre bande du caryotype. Parmi ces autres bandes, certaines sont impliquées aussi de façon récurrente comme les bandes 2pir, 2pi2, 22qII. 2 et 22qI3.2 (figure 3, p. 30I). Enfin, environ $4 \%$ des mitoses sont porteuses d'un remaniement impliquant n'importe quels autres chromosomes, sans que l'on puisse déceler la moindre spécificité.

Ces différentes catégories de remaniements peuvent être observées dans les lymphocytes de sujets sains, mais avec une très faible fréquence. C'est ainsi que les remaniements des chromosomes 7 et 14 sont observés dans environ $0,2 \%$ des lymphocytes de sujets témoins, soit 30 fois moins fréquemment que chez les sujets AT.

\section{Nature de ces remaniements}

La très grande récurrence des remaniements impliquant les chromosomes 7 et 14 chez les sujets AT et, dans une moindre mesure, chez les sujets témoins n'a reçu que très récemment un début d'explication. Il est en eff et apparu clairement au cours de l'année 1985 qu'il existait une étroite corrélation entre les points de remaniements des chromosomes 2, 7, 14 et 22 et les localisations chromosomiques des gènes de la superfamille des immunoglobulines (tableau I). Les gènes de cette famille, qui codent pour les 


\section{REFERENCES}

I. Hecht F, Koler RO, Rigas DA, et al. Leukemia and lymphocytes in AT. Lancet 1966; ii: 1193.

2. Taylor AMR. Cytogenetics of amxia telangiectasia. In: Bridges BA, Harnden DG, eds. Ataxia Telangiectasia. Chichester: John Wilcy and Sons, 1982: 53-81.

3. Aurias A, Dutrillaux B, Buriot D, Lejeune J. High frequency of inversions and translocations of chromosome 7 and 14 in ataxia telangicctasia. Mutat Res 1980; 69: 369-74.

4. Aurias A, Couturier J, Dutrillaux AM, et al. Inversion (14) (q12 qter) or (q11 . 2 932.3): the most frequently acquired rearrangement in lymphocytes. Hum Genet 1985; 71 : 19-21.

5. Baer R, Chen KC, Smith SD, Rabbitts TH Fusion of an immunoglobulin variable gene and a $\mathrm{T}$ cell receptor constant gene in the chromosome 14 inversion associated with T cell tumors. Cell 1985; 43: 705-13.

6. Aurias A, Croquette MF, Nuyts JP, Griscelli C, Dutrillaux B. New data on clonal anomalies of chromosome 14 in ataxia telangiectasia: tct (14 14) and inv (14). Hum Genet 1986; $72: 22-4$.

7. Johnson J, Gatti RA, Cavenec W, Drayna D, White R. Proc 17 th Miami Winter Symposium 1985; 2.

8. Kaiser-Mc Caw B, Hecht F. Ataxia telangiectasia: chromosomes and cancer. In : Bridges B A Harnden D G, eds. Ataxia Telangiectasia. Chichester: John Wiley and Sons, 1982: 243-57.

Figure 2. Remaniements sporadiques récurrents des chromosomes 7 et 14. Les chromosomes normaux sont ceux pour lesquels /e numéro a été précisé. Les flèches indiquent les points d'échange de matérie chromosomique. Tous ces remaniements correspondent à des échanges entre les 4 bandes indiquées à la figure 1. Marquage des chromosomes en



$m / s n^{0} 6$, vol. 2, juin-juilles 
chaines d'immunoglobulines, les chaînes du récepteur des lymphocytes $T$ et divers antigènes de différenciation des lymphocytes, présentent entre eux de grandes analogies de structure et de séquence (voir l'article de B. Malissen dans ce numéro). La majorité de ces gènes subit, au cours de la maturation des lymphocytes, différentes recombinaisons somatiques qui aboutissent à la formation de gènes simplifiés fonctionnels. Normalement, ces recombinaisons somatiques impliquent les éléments d'un gène donné, ce qui n'entraîne aucune modification du caryotype décelable avec les techniques classiques de cytogénétique. Il est très probable que les remaniements des chromosomes 2, 7, 14 et 22 que nous observons correspondent en fait à des recombinaisons illégitimes entre des gènes différents de la superfamille. Ainsi, par exemple, l'inversion du chromosome 7, si fréquente dans l'ataxie télangiectasie, pourrait correspondre à une recombinaison illégitime entre les gènes $\beta$ et $\gamma$ du récepteur $T$, et l'inversion du chromosome ${ }_{14}$, relativement fréquente dans la population générale, à une recombinaison illégitime entre le gène $\alpha$ du récepteur $T$ et le gène $H$ de la chaîne lourde des immunoglobulines. Ces remaniements illégitimes surviennent apparemment chez n'importe quel sujet de la population générale [4] et même dans d'autres espèces puisque nous en avons observé chez le chimpanzé. Leur très grande fréquence dans l'ataxie télangiectasie pourrait s'expliquer soit par une fréquence accrue de formation, et dans ce cas nous pourrions penser que les enfants ataxiques souffrent d'un trouble de la recombinaison des gènes de la superfamille, soit par un avantage sélectif accru des cellules porteuses de tels remaniements chez l'enfant ataxique. En faveur de cette dernière hypothèse, nous pouvons remarquer que les fréquences relatives de ces différents remaniements ne sont pas du tout semblables dans la population générale et chez les sujets AT. Remarquons aussi qu'une inversion du chromosome I4 apparemment identique $\grave{a}$ celle observée avec une faible fréquence dans les lymphocytes de sujets sains est retrouvée dans des clones de leucémies ou de lymphomes $T$. Dans un tel lymphome $T$, il vient d'être démontré que le remaniement du chromosome 14 correspondait à une fusion illégitime entre une partie variable d'un gène de chaîne lourde d'immunoglobuline et une partie constante d'un gène $\alpha$ du récepteur $T$ [5]. Le gène de fusion ainsi constitué est fonctionnel et l'on peut penser que son expression est en rapport étroit avec l'avantage sélectif qui a été acquis par les cellules tumorales.

\section{Second stade évolutif}

Chez environ $10 \%$ des patients, une importante population lymphocytaire porteuse d'un remaniement chromosomique unique est détectée. La survenue de ce clone ne s'accompagne d'aucun signe clinique ou biologique particulier et seul l'examen chromosomique permet de le déceler. Notons que l'apparition de ces clones semble corrélée avec l'âge des patients puisqu'on les observe chez des malades dont l'âge moyen est de 13 ans. Lorsque des examens successifs ont pu être réalisés chez un même patient, il est habituel de constater un accroissement progressif de la fréquence des cellules porteuses du remaniement clonal et ce remaniement peut, en quelques années, être observé dans pratiquement $100 \%$ des lymphocytes stimulés par la PHA. En revanche, il n'est jamais retrouvé dans les lymphocytes stimulés par le PWM et il semble qu'il soit limité à une population de lymphocytes exprimant l'antigène $\mathrm{T}_{4}$. Notons encore que les mitoses qui ne portent pas ce remaniement clonal présentent exactement les mêmes remaniements sporadiques que ceux observés lors du premier stade évolutif et que, dans une même mitose, on n'observe jamais d'association entre remaniement clonal et remaniement sporadique des chromosomes 7 et/ou I4.

Ces remaniements clonaux ont pour particularité de toujours impliquer la partie proximale du bras long du chromosome i4. Les plus fréquents d'entre eux sont des inversions du chromosome I4 et des transloca-

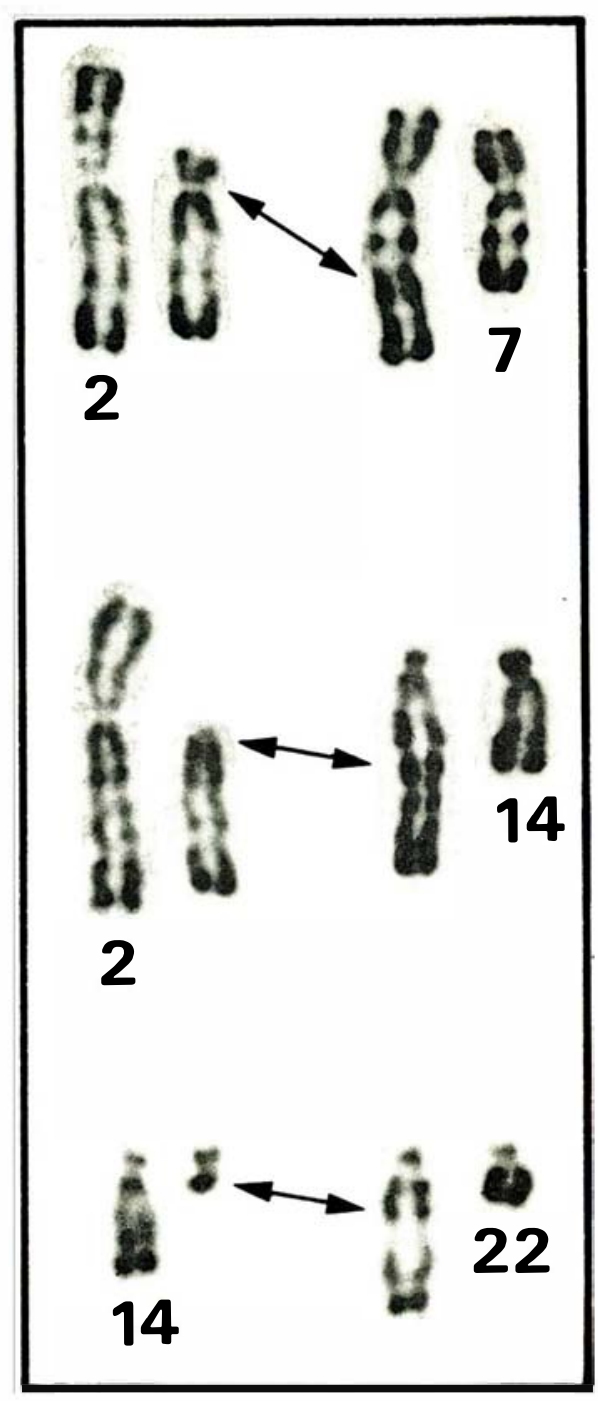

Figure 3. Remaniements entre les chromosomes 7 ou 14 et les chromosomes 2 ou 22. En haut, translocation entre les bandes $2 p 12$ et 7q35. Au milieu, translocation entre les bandes $2 p 11$ et 14qter. En bas, translocation entre les bandes $14 q 12$ et $22 q 12$. 
tions entre les deux chromosomes 14. Bien que ces remaniements ressemblent grossièrement aux remaniements sporadiques des chromosomes I4 que nous avons décrits précédemment lors du premier stade évolutif, une analyse minutieuse des points de cassure avec des techniques de marquage à haute résolution montre qu'il n'en est rien et qu'au moins l'un des points est différent (figure 4). D'une façon générale, il semble que ces remaniements clonaux du chromosome I4 impliquent les bandes I4qI I.2 et I 4932. I, avec dans certains cas un remaniement plus complexe qui pourrait correspondre à une duplication inversée du segment I4932. I à I4qter [6]. Si le point de cassure en I49II.2 peut éventuellement être le même que celui observé dans les remaniements sporadiques et dans les leucémies ou lymphomes $T$, c'est-à-dire le gène $\alpha$ du récepteur T, il n'en est pas de même pour le point situé en I4932. I qui est clairement plus proximal dans les clones d'ataxie et qui ne devrait pas impliquer le gène $\mathrm{H}$ des immunoglobulines. La nature du gène ainsi remanié en I4932. I demeure pour l'instant inconnue. Nous pouvons cependant penser que sa translocation avec le locus $\alpha$ donne à la cellule un avantage sélectif important, sans pour autant lui conférer un caractère tumoral. Une approche moléculaire de la nature de ces remaniements est actuellement en cours dans différents laboratoires. Le seul résultat actuellement publié fait état d'une duplication inversée centrée sur une région $\mathrm{J}$ du locus $\mathrm{H}$ des immunoglobulines dans un cas de translocation entre les deux chromosomes 14 [7]. Cette duplication inversée pourrait correspondre à l'image cytogénétique complexe de duplication inversée du segment I4932. I-I4qter que nous avons signalée précédemment. Une éven-

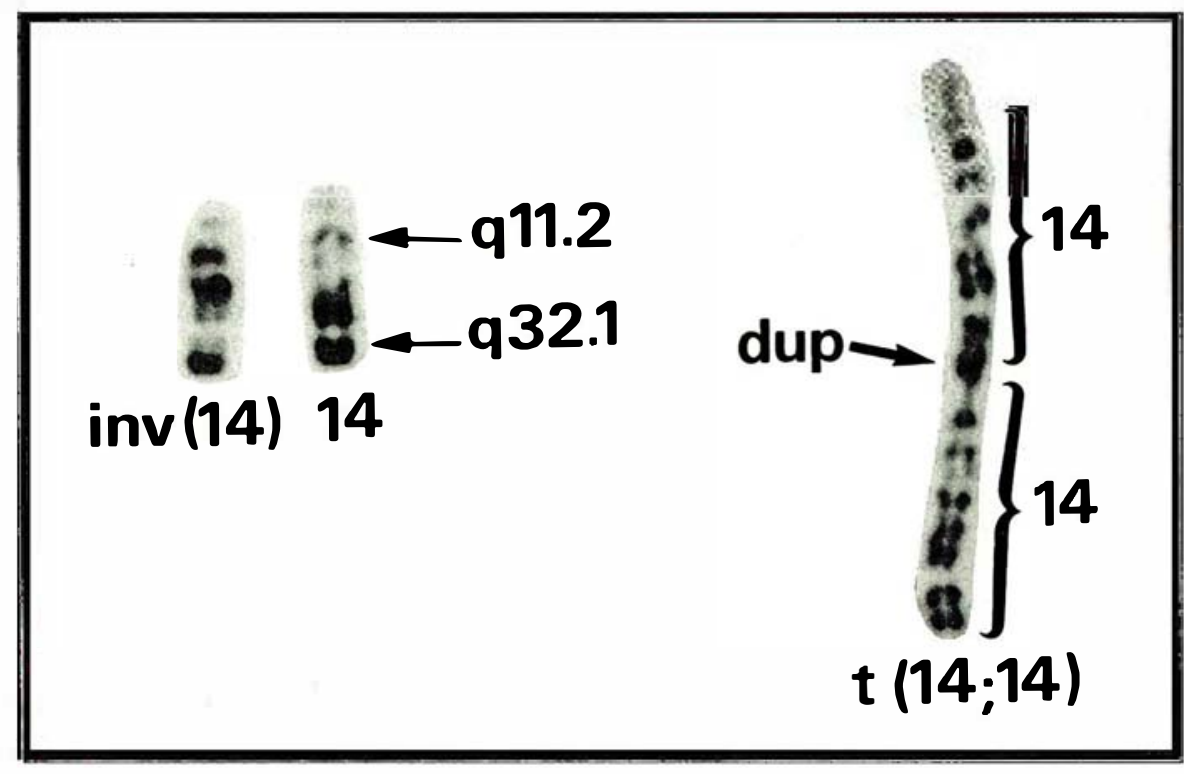

Figure 4. Remaniements clonaux des chromosomes 14. A gauche, inversion du chromosome 14. A droite, translocation entre les deux chromosomes 14. Les points de cassure en 14q11.2 et 14q32.1 sont indiqués sur le chromosome 14 normal. La flèche "dup" indique la probable duplication du segment q32. 1 -qter dans cette translocation $t(14 ; 14)$. Marquage en bandes $R$ à haute résolution. tuelle relation de causalité entre les remaniements sporadiques préexistants et ces remaniements clonaux n'est pas démontrée.

\section{Troisième stade évolutif}

Chez certains enfants AT, porteurs connus d'un clone impliquant le chromosome I4, une leucémie lymphoïde chronique $\mathrm{T}$ va débuter [8]. Cette leucémie se développe à partir des cellules ayant le remaniement clonal. Cependant, à ce stade de l'évolution, d'autres aberrations chromosomiques sont observées en plus du remaniement clonal initial, et les responsabilités relatives de ces différentes aberrations dans le processus de cancérisation sont encore difficiles à préciser.

\section{Le problème des hétérozygotes}

Nous avons signalé que l'incidence de la maladie était d'environ I/40000. Si cette affection était la conséquence d'une mutation unique, nous attendrions donc environ I \% d'hétérozygotes dans la population générale. Cependant, l'existence d'au moins 4 groupes de complémentation est maintenant établie, et la fréquence des hétérozygotes pourrait donc être sensiblement plus importante.

Il semble que ces hétérozygotes présentent a minima différentes manifestations de la maladie, comme un risque accru de cancers, une radiosensibilité augmentée et des remaniements chromosomiques sporadiques. C'est ainsi qu'environ I \% des lymphocytes d'hétérozygotes obligatoires sont porteurs d'un remaniement des chromosomes 7 et/ou I4. Ce taux, bien que nettement supérieur à celui de la population générale, est cependant trop faible pour permettre un diagnostic cytogénétique de l'état hétérozygote. Il en est de même pour les autres tests biologiques et le diagnostic de l'état hétérozygote apparaît encore comme très aléatoire. Une telle possibilité serait pourtant précieuse, d'une part pour effectuer un conseil génétique dans les fratries d'enfants atteints et d'autre part pour aborder le véritable pro- 
blème épidémiologique posé par ces hétérozygotes. Une solution satisfaisante ne sera vraisemblablement trouvée que le jour où les gènes de l'ataxie télangiectasie seront identifiés. Différentes équipes travaillent actuellement à cette identification en étudiant dans les familles AT la coségrégation du gène $A T$ et de différents marqueurs polymorphiques dont la localisation chromosomique est connue.

\section{Conclusion}

L'ataxie télangiectasie est ainsi l'une des rares maladies où la cytogénétique permet de suivre les différentes étapes d'un processus de cancérisation. L'étroite intrication des facteurs immunologiques et cytogénétiques, la probable implication des gènes de la superfamille des immunoglobulines font de cette affection un modèle passionnant de maladie précancéreuse.

D'autre part, la grande fréquence des hétérozygotes dans la population générale, leur radiosensibilité augmentée et leur risque accru de cancer rendraient très souhaitable leur dépistage et une surveillance médicale régulière. Ceci ne sera sans doute possible que lorsque les gènes de l'ataxie télangiectasie seront clairement identifiés et clonés

\section{Summary}

Ataxia telangiectasia (AT) is a rare genetic disorder with autosomal recessive inheritance. Cerebellar ataxia, telangiectasia and recurrent infections are common features of the disease. Cancer susceptibility and radiation sensitivity are also well documented. Typical chromosomal aberrations observed in AT lymphocytes are reported here. In about $90 \%$ of AT patients, around $6 \%$ of the lymphocytes carry an aberration involving chromosomes 7, I4, 2 and/or 22. These aberrations probably correspond to illegitimate rearrangements of immunoglobulin superfamily genes. In the other $10 \%$ of the patients, in addition to the anomalies previously quoted, large precancerous clones involving band qII. 2 of chromosome I4 are observed. Patients with such clones tend to develop chronic $\mathrm{T}$ cell leukemia. Cancer susceptibility, radiation sensitivity and chromosomal aberrations are observed, to a lesser degree, in AT heterozygotes.

\section{* GLOSSAIRE*}

Maladies cassantes : un certain nombre de maladies s'accompagnent d'un excès de cassures chromosomiques. Trois d'entre elles répondent à un déterminisme génétique récessif autosomique : l'ataxie télangiectasie, le syndrome de Bloom et l'anémie de Fanconi. Pour ces trois maladies, un défaut des systèmes de réparation de $\lceil A D N$ est évoqué et ces maladies sont parfois intitulées réparatoses. Les autres maladies cassantes sont des affections autoimmunes, comme la sclérodermie ou le lupus. Translocation : il s'agit d'un échange de matériel entre deux chromosomes différents.

Inversion péricentrique : ce remaniement chromosomique cor- respond à un échange de segments terminaux entre le bras court et le bras long d'un même chromosome, ce qui est l'équivalent d'une rotation (inversion) du segment central (péricentrique).

Nomenclature : pour chaque chromosome, on définit un bras court $p$ et un bras long $q$. Chaque bande est caractérisée par un numéro : 7PI 4 signifie qu'il s'agit de la bande 14 du bras court $p$ du chromosome 7. Une translocation est caractérisée par un " $t$ " suivi d'une parenthèse où figurent les deux chromosomes ayant échangé des segments. De même, une inversion se définit par "inv" suivi d'une parenthèse où figure le chromosome inversé. 